\title{
A replication study confirms the association of TNFSF4 (OX40L) polymorphisms with Systemic Sclerosis in a large European cohort
}

\author{
L Bossini-Castillo ${ }^{1}$, JCA Broen ${ }^{2}$, C P Simeon ${ }^{3}$, L Beretta ${ }^{4}$, M C Vonk ${ }^{2}$, N Ortego-Centeno ${ }^{5}$, G Espinosa ${ }^{6}$, P Carreira ${ }^{7}$, \\ M T Camps ${ }^{8}, N_{\text {Navarrete }}^{9}$, M F González-Escribano ${ }^{10}$, E Vicente-Rabaneda $^{11}$, L Rodríguez $^{12}$, C Tolosa $^{13}$, \\ J A Román-Ivorra ${ }^{14}$, I Gómez-Gracia ${ }^{15}$, F J García-Hernández ${ }^{16}$, I Castellvi ${ }^{17}$, M Gallego ${ }^{18}$, A Fernández-Nebro ${ }^{19}$, \\ M V Egurbide ${ }^{20}$, V Follonosa ${ }^{3}$, P García de la Peña ${ }^{21}$, A Pros ${ }^{22}$, M A González-Gay ${ }^{23}$, R Hesselstrand ${ }^{24}$, \\ G Riemekasten ${ }^{25}$, T Witte ${ }^{26}$, MJH Coenen ${ }^{27}$, B P Koeleman ${ }^{28}$, F Houssiau ${ }^{29}$, V Smith ${ }^{30}$, F De Keyser ${ }^{30}$, R Westhovens ${ }^{31}$, \\ E De Langhe ${ }^{31}$, A E Voskuyl ${ }^{32}$, A J Schuerwegh ${ }^{33}$, M M Chee $^{34}$, R Madhok $^{34}$, P Shiels ${ }^{34}$, C Fonseca $^{35}$, C Denton ${ }^{35}$, \\ K Claes ${ }^{36}$, L Padykov ${ }^{37}$, A Nordin ${ }^{37}, \varnothing$ Palm ${ }^{38}$, B A Lie ${ }^{39}$, P Airó ${ }^{40}$, R Scorza ${ }^{4}$, J M van Laar ${ }^{41}$, N Hunzelmann ${ }^{42}$, \\ A Kreuter $^{43}$, A Herrick ${ }^{44}$, J Worthington ${ }^{44}$, TRD J Radstake ${ }^{2}$, J Martín ${ }^{1 \dagger}$, B Rueda $^{1,10^{*+}}$ \\ From 5th European Workshop on Immune-Mediated Inflammatory Diseases \\ Sitges-Barcelona, Spain. 1-3 December 2010
}

\section{Introduction}

The TNFSF4 gene, which encodes OX40L, is considered as a potential autoimmunity candidate gene. OX40L is expressed on activated antigen presenting cells (APCs) and endothelial cells in acute inflammation [1]. Furthermore, it enhances B-cell proliferation and differentiation, and its binding to OX40 (CD134) promotes proliferation and survival of $\mathrm{T}$-cells and predisposes them to a more permissive proliferative and survival condition [2]. Interestingly, four TNFSF4 promoter single-nucleotide polymorphisms (SNP) were recently implicated in susceptibility to systemic sclerosis (SSc) [3].

\section{Aim}

The aim of this study was to confirm the influence of TNFSF4 polymorphisms on SSc susceptibility and clinical subtypes or phenotypic features.

\section{Patients and methods}

Eight European populations of Caucasian ancestry were included, comprising a total of 3014 SSc patients and 3125 healthy controls. Four genetic variants of the TNFSF4. gene (rs1234314, rs844644, rs844648 and rs12039904)

+ Contributed equally

${ }^{1}$ Instituto de Parasitología y Biomedicina López-Neyra, CSIC, Granada, Spain Full list of author information is available at the end of the article were selected as genetic markers and genotyped using Taqman Allelic Discrimination Assays.

\section{Results}

A pooled-analysis revealed the association of rs 1234314 and rs12039904 SNPs with SSc [OR=1.15,95\%CI 1.02$1.31 ; \mathrm{OR}=1.18,95 \% \mathrm{CI} 1.08-1.29$, respectively].

After subtype stratification, significant association of the four tested SNPs with the limited cutaneous SSc (lcSSc) subgroup of patients was revealed [rs1234314 $\mathrm{OR}=1.22,95 \% \mathrm{CI} 1.07-1.38 ;$ rs $844644 \mathrm{OR}=0.91,95 \% \mathrm{CI}$ 0.83-0.99; rs844648 OR=1.10,95\%CI 1.01-1.20; and rs12039904 OR=1.20,95\%CI 1.09-1.33]. Considering autoantibody status, the association of three of these variants, rs1234314, rs844648 and rs12039904 with anticentromere autoantibody (ACA) positive subset of patients remained significant $[\mathrm{OR}=1.23,95 \% \mathrm{CI} 1.10-1.37$; $\mathrm{OR}=1.12,95 \% \mathrm{CI} 1.01-1.25$; OR=1.22,95\%CI $1.07-1.38$, respectively]. Haplotype analysis confirmed the existence of a previously described protective haplotype and revealed a new risk haplotype with evidence of association with SSc [OR=0.88,95\%CI $0.82-0.96$; OR $=1.14,95 \% \mathrm{CI}$ $1.03-1.26$, respectively], lcSSc [OR $=0.88,95 \% \mathrm{CI}$ 0.80-0.96; $\mathrm{OR}=1.20,95 \% \mathrm{CI} 1.08-1.35$, respectively] and ACA positive subgroups[OR=0.86,95\% CI $0.77-0.97 ; \mathrm{OR}=1.23,95 \%$ CI 1.07-1.42, respectively]. 


\section{Conclusions}

Our data confirm the influence of TNFSF4 polymorphisms in SSc genetic susceptibility, especially with lcSSc and ACA positive subsets of patients.

\begin{abstract}
Author details
${ }^{1}$ Instituto de Parasitología y Biomedicina López-Neyra, CSIC, Granada, Spain.

${ }^{2}$ Dept. of Rheumatology, Radboud University Nijmegen Medical Centre, Nijmegen, The Netherlands. ${ }^{3}$ Servicio de Medicina Interna, Hospital Valle de Hebron, Barcelona, Spain. ${ }^{4}$ Referral Center for Systemic Autoimmune Diseases, University of Milan, Milan, Italy. ${ }^{5}$ Servicio de Medicina Interna, Hospital Clínico Universitario, Granada, Spain. ${ }^{6}$ Servicio de Medicina Interna, Hospital Clínico de Barcelona, Barcelona, Spain. ' Servicio de Reumatología, Hospital 12 de Octubre, Madrid, Spain. ${ }^{8}$ Servicio de Medicina Interna, Hospital Carlos-Haya, Málaga, Spain. ${ }^{9}$ Servicio de Medicina Interna, Hospital Virgen de las Nieves, Granada, Spain. ${ }^{10}$ Servicio de Inmunología, Hospital Virgen del Rocío, Sevilla, Spain. ${ }^{11}$ Servicio de Reumatología, Hospital de la Princesa, Madrid, Spain.

${ }^{12}$ Servicio de Reumatología, Hospital Clinico San Carlos, Madrid, Spain.

${ }^{13}$ Servicio de Medicina Interna, Hospital Parc Tauli,Sabadell, Spain. ${ }^{14}$ Servicio de Reumatología, Hospital del Doctor Peset Aleixandre, Valencia, Spain.

${ }^{15}$ Servicio de Reumatología, Hospital Reina Sofía, Córdoba, Spain. ${ }^{16}$ Servicio de Medicina Interna, Hospital Virgen del Rocío, Sevilla, Spain. ${ }^{17}$ Servicio de Reumatología, Hospital de Sant Pau, Barcelona, Spain. ${ }^{18}$ Servicio de Medicina Interna, Hospital Central de Asturias, Oviedo, Spain. ${ }^{19}$ Servicio de Reumatología, Hospital Carlos Haya, Málaga, Spain. ${ }^{20}$ Servicio de Medicina Interna, Hospital de Cruces, Barakaldo, Spain. ${ }^{21}$ Servicio de Reumatología, Hospital Ramón y Cajal, Madrid, Spain. ${ }^{22}$ Servicio de Reumatología, Hospital Del Mar, Barcelona, Spain. ${ }^{23}$ Servicio de Reumatología, Hospital Universitario Marqués de Valdecilla, Santander, Spain. ${ }^{24}$ Dept. of Rheumatology, Lund University Hospital, Lund, Sweden. ${ }^{25}$ Dept. of Rheumatology and Clinical Immunology, Charité University Hospital, Berlin, Germany. ${ }^{26}$ Hannover Medical School, Hannover, Germany. ${ }^{27}$ Dept. of Human Genetics, Radboud University Nijmegen Medical Centre, Nijmegen, The Netherlands. ${ }^{28}$ Section Complex Genetics, Dept. of Medical Genetics, University Medical Center Utrecht, Utrecht, The Netherlands. ${ }^{29}$ Université catholique de Louvain (UCL), Brussels, Belgium. ${ }^{30}$ University of Ghent, Ghent, Belgium. ${ }^{31}$ University of Leuven (KULeuven), Leuven, Belgium. ${ }^{32}$ Dept. of Rheumatology, VU University Medical Center, Amsterdam, The Netherlands. ${ }^{33}$ Dept. of Rheumatology, Leiden University Medical Center, Leiden, The Netherlands. ${ }^{34}$ University of Glasgow, Glasgow, UK. ${ }^{35}$ Centre for Rheumatology, Royal Free and University College Medical School, London, UK. ${ }^{36}$ Dept. of Genetics, University of Ghent, Ghent, Belgium. ${ }^{37}$ Karolinska Institute, Stockholm, Sweden. ${ }^{38}$ Dept. of Rheumatology, Rikshospitalet, Oslo University Hospital, Oslo, Norway. ${ }^{39}$ Institute of Immunology, Rikshospitalet, Oslo University Hospital, Oslo, Norway. ${ }^{40}$ Servizio di Reumatologia ed Immunologia Clinica Spedali Civili, Brescia, Italy. ${ }^{41}$ Institute of Cellular Medicine, Newcastle University, Newcastle, UK ${ }^{42}$ Dept. of Dermatology, University of Cologne, Cologne, Germany. ${ }^{43}$ Ruhr University of Bochum, Bochum, Germany. ${ }^{44}$ Arthritis Research UK Epidemiology Unit, The University of Manchester, Manchester Academic Health Science Centre, Manchester, UK.
\end{abstract}

Published: 25 November 2010

\section{References}

1. Manku H, Graham DS, Vyse TJ: Association of the co-stimulator OX40L with systemic lupus erythematosus. J Mol Med. 2009, 87:229-234.

2. Gough MJ, Weinberg AD: OX40 (CD134) and OX40L. Adv Exp Med Biol. 2009, 647:94-107.

3. Gourh P, Arnett FC, Tan FK, Assassi S, Divecha D, Paz G, et al: Association of TNFSF4 (OX40L) polymorphisms with susceptibility to systemic sclerosis. Ann Rheum Dis. 2010, 69:550-555.

doi:10.1186/1479-5876-8-S1-P5

Cite this article as: Bossini-Castillo et al: A replication study confirms the association of TNFSF4 (OX40L) polymorphisms with Systemic Sclerosis in a large European cohort. Journal of Translational Medicine 2010 8(Suppl 1):P5.

\section{Submit your next manuscript to BioMed Central and take full advantage of:}

- Convenient online submission

- Thorough peer review

- No space constraints or color figure charges

- Immediate publication on acceptance

- Inclusion in PubMed, CAS, Scopus and Google Scholar

- Research which is freely available for redistribution

Submit your manuscript at www.biomedcentral.com/submit
Biomed Central 